\title{
Incidencia de las Prácticas de Gestión del Conocimiento sobre la Creatividad Organizacional
}

\author{
Andrea Echeverri, Nelson Lozada* y José E. Arias \\ Universidad de Antioquia, Facultad de Ciencias Económicas, Depto. de Administración de Empresas, \\ Calle 67 No. 53-108 oficina 13-414 Medellín-Colombia. (e-mail:nelson.lozada@udea.edu) \\ ${ }^{*}$ Autor a quien debe dirigirse la correspondencia.
}

Recibido May. 17, 2017; Aceptado Jul. 20, 2017; Versión final Oct. 25, 2017, Publicado Feb. 2018

\begin{abstract}
Resumen
El presente artículo estudia la manera como el conocimiento puede ser gestionado dentro de las organizaciones para promover la generación de ideas nuevas y útiles. El objetivo del artículo es evidenciar las prácticas concretas de gestión del conocimiento que tienen incidencia sobre la creatividad organizacional. En lo metodológico se utilizaron ecuaciones estructurales por el método mínimos cuadrados parciales y el análisis bootstrap-percentil para probar la existencia de la mediación en una muestra de 160 empresas. Se encontró que las prácticas de aprendizaje continuo y la gestión de las competencias individuales de los empleados, tienen incidencia significativa sobre la creatividad, considerada una fuente de ventaja competitiva y un elemento indispensable para la supervivencia de las organizaciones. Por lo tanto, se reconoce que la puesta en marcha de prácticas de gestión del conocimiento fomenta el ambiente organizacional, proporciona recursos, y desarrolla procesos que promueven la creatividad organizacional y facilitan el proceso de innovación.
\end{abstract}

Palabras clave: conocimiento organizacional, gestión del conocimiento, creatividad organizacional, aprendizaje continuo, gestión de competencias

\section{Impact of Knowledge Management Practices on Organizational Creativity}

\begin{abstract}
This paper studies how knowledge can be managed within the organizations to promote the generation of new and useful ideas. The aim of the paper is to show specific knowledge management practices that have impact on organizational creativity. Structural equations by the partial least squares method and bootstrappercentile analysis to test the existence of mediation in a sample of 160 companies were used. It was found that continuous learning practices and management of individual skills of employees have significant impact on creativity, considered a source of competitive advantage and an indispensable element for the survival of organizations. Therefore, it was recognized that startup of knowledge management practices promotes an organizational environment, provides resources, and develops processes that promote organizational creativity and facilitate the process of innovation.
\end{abstract}

Key words: organizational knowledge, knowledge management, organizational creativity, continuous learning, management skills 


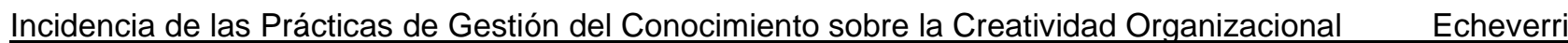

\section{INTRODUCCIÓN}

La velocidad de los cambios y las presiones de la actual economía globalizada, obligan a las organizaciones a desarrollar iniciativas que les permitan mejorar la respuesta a los procesos cada vez más dinámicos y brindar soluciones creativas a los problemas del entorno (Davenport y Prusak, 1998). En este contexto, se considera al conocimiento como un recurso fundamental, que debe ser evaluado y controlado para maximizar su productividad (Alavi y Leidner, 2001). Desde este punto de vista, el éxito de la Gestión del Conocimiento (GC) está asociado con que los nuevos conocimientos mejoren las acciones organizativas o creen la base para una nueva acción, de manera que faciliten el rendimiento creativo y dirijan el conjunto de conocimientos de la empresa hacia la generación de ideas nuevas y útiles (Amabile, 1988).

En la literatura existente, la creatividad organizacional es considerada un resultado intermedio que se relaciona positivamente con la creación de conocimiento y el desempeño organizacional, lo que sugiere que puede favorecer la eficacia y la supervivencia de las organizaciones, al ser punto de partida de la innovación organizacional y un elemento que transforma el conocimiento en valor para el negocio (Lee y Choi, 2003). Zhou y George (2001) señalan que es fundamental un entorno organizativo en el que se reconozcan, promuevan y recompencen las ideas de los empleados, de manera que se fomente la generación de ideas nuevas y útiles. Así mismo, el desarrollo de una cultura de colaboración que permita la adquisición y el intercambio de conocimientos y experiencias. Las actividades relacionadas con la retroalimentación, facilitan también la generación de ideas creativas, en la medida que dirigen la atención de los empleados hacia la búsqueda de nuevas formas de hacer las cosas, mejoran la orientación a la tarea, y pueden tener una mayor disposión para el aprendizaje y el desarrollo (Zhou y George, 2001).

A pesar de la relevancia que ha sido ampliamente reconocida de la gestión del conocimiento y la creatividad organizacional, existe una carencia de trabajos que estudien su papel potencial las relaciones existentes entre ambas, las influencias del entorno de trabajo y la forma en que pueden ser maximizadas para garantizar la generación y aplicación de ideas nuevas y útiles. La mayoría de trabajos ofrecen un análisis muy amplio que no logra esclarecer las relaciones causales entre la prácticas de gestión del conocimiento y la creatividad organizacional (Phipps y Prieto, 2012; Sigala y Chalkiti, 2015; Rhee y Choi, 2017). La mayoría de ellos bajo la perspectiva de los tipos de conocimiento, explícito y tácito, propuesto por Polanyi (1966) y ampliamente desarrollado por Nonaka (Nonaka, 1994). Si bien la distinción entre conocimiento explícito y tácito, ofrece una comprensión acerca de la manera como se crea y se puede controlar el conocimiento en las organizaciones (Nonaka, 1994), no reconoce las actividades especificas que pueden ejecutar las organizaciones para mejorar el proceso creativo. Por lo tanto, el presente trabajo tiene como propósito evidenciar las prácticas concretas de GC que influencian la creatividad organizacional, y reconocer la manera de maximizar el proceso creativo, considerado el primer paso para los procesos de innovación dentro de las organizaciones. Para ello, se utilizaron ecuaciones estructurales por el método mínimos cuadrados parciales y el análisis bootstrappercentil para probar la existencia de la mediación en una muestra de 160 empresas.

\section{LA CREATIVIDAD ORGANIZACIONAL}

La creatividad organizacional es definida como la generación de ideas nuevas y originales, y potencialmente relevantes o útiles en relación con nuevos productos, servicios y procesos organizativos (Amabile, 1988; Amabile y Conti, 1999). Es a menudo considerada el primer paso para la innovación organizacional (Zhou y George, 2001), entendida esta ultima como la aplicación o implementación exitosa de ideas creativas por parte de la organización (Van de Ven, 1986). Ambas se han convertido en una fuente de ventaja competitiva distinta, que contribuye al desempeño, el éxito y la supervivencia a largo plazo de las organizaciones (Anderson et al., 2014) en el entorno empresarial turbulento y competitivo de hoy que obliga a las organizaciones a innovar constantemente (Amabile, 1988). Por lo tanto, es cada vez más importante para las organizaciones determinar los factores que promueven la creatividad organizacional y fomentan la visión creativa de los individuos, de manera que puedan aprovechar los beneficios de su originalidad e inventiva (Phipps y Prieto, 2012).

De acuerdo con Amabile y Conti (1999) se consideran cinco componentes del ambiente organizacional que afectan la creatividad: el fomento de la creatividad, que comprende el apoyo que se le da desde todos los niveles organizativos a nuevas ideas y el flujo de información; los recursos, es decir, los materiales, información y recursos generales disponibles para el trabajo incluyendo las finanzas, la disponibilidad de tiempo y recursos de personal (Anderson et al., 2014); las presiones tanto positivas como negativas; los impedimentos organizacionales a la creatividad y la autonomía o libertad de trabajo. Esta perspectiva se conoce como la teoría componencial de la creatividad y se basa en la idea de que los entornos de trabajo tienen un impacto sobre la creatividad (Amabile, 1997). Otra importante perspectiva se basa en las implicaciones de las diferencias culturales sobre la creatividad (Zhou y George, 2001). Considera las diferencias culturales en la creatividad individual, la manera como las tareas y los contextos sociales moderan 
la relación entre los valores culturales de los individuos y la creatividad, por ejemplo, factores como el individualismo, el control de la incertidumbre o la estructura jerárquica (Anderson et al., 2014). En cuanto a la creatividad de equipo, sugiere que las diferencias culturales pueden incidir en la producción de ideas creativas de equipos integrados de trabajo.

\section{EL CONOCIMIENTO ORGANIZACIONAL}

El conocimiento es reconocido como un recurso fundamental de la sociedad moderna y las organizaciones (Alegre, 2004; Adelstein y Clegg, 2014). A diferencia de los recursos tradicionales de capital, tierra y trabajo, tiene un potencial ilimitado para el crecimiento de la organización, debido a que provee una ventaja competitiva sostenible y genera rendimientos crecientes (Grant, 1996; Lee et al., 2016). "El conocimiento es una mezcla fluida de experiencia enmarcada, valores, información contextual, y visión de expertos que proporciona un marco para evaluar e incorporar nuevas experiencias e información" (Davenport y Prusak, 1998). Desde este punto de vista, si bien el conocimiento es originado en la mente de las personas, el conocimiento organizacional se produce cuando sus miembros comparten creencias e interactúan para trabajar juntos. Por lo tanto, se encuentra incorporado en diferentes ámbitos de la organización, en la cultura, identidad, prácticas, documentos, rutinas, políticas, procedimientos, sistemas, normas, así como en los empleados (Alavi y Leidner, 2001; García-Quevedo et al., 2013).

De acuerdo con la literatura de la gestión estratégica una cuestión clave consiste en la explicación de las diferencias en los resultados empresariales. La teoría basada en los recursos ofrece una interpretación esclarecedora sobre este fenómeno: la posesión de recursos escasos, valiosos, inimitables y difíciles de sustituir, implica que algunas empresas obtengan mejores resultados de manera prolongada en el tiempo (Barney, 1991). A su vez, el enfoque basado en el conocimiento, sostiene que los recursos basados en el conocimiento al ser socialmente complejos y difíciles de imitar, pueden producir una ventaja competitiva sostenible a largo plazo (Von Krogh et al., 2000; Vásquez-Rizo et al., 2015; Lee et al., 2016). No obstante, no es el conocimiento por sí mismo la base para lograr una ventaja competitiva, sino la capacidad de la empresa para controlar y aplicar eficazmente los conocimientos adquiridos, desarrollar sistemas y procedimientos adecuados para gestionar y aumentar los conocimientos, y las habilidades para incorporarlos y transformarlos en beneficio de la organización (Phipps y Prieto, 2012; Shahzad et al., 2016).

La GC se ha convertido en un valor clave para las organizaciones (Donate y Sánchez de Pablo, 2015). Esta se refiere al conjunto de actividades, iniciativas y estrategias utilizadas en una organización para administrar el conocimiento (Alavi y Leidner, 2001). Es un enfoque sistemático y organizado que le permite a las organizaciones ser más rápidas, eficientes, innovadoras y eficaces que la competencia (Shahzad et al., 2016). El modelo SECI (Nonaka y Takeuchi, 1995) es bien conocido dentro de las teorías de la GC y se deriva de la distinción entre las dimensiones explícita y tácita del conocimiento (Polanyi, 1966). El conocimiento explícito es aquel que puede ser codificado y almacenado, y es fácil de transmitir a otros a través de lenguaje formal o de forma simbólica (Von Krogh et al., 2000) tales como bases de datos, archivos, reglas y fórmulas. Por su parte, el conocimiento tácito sugiere una cualidad personal que lo hace difícil de formalizar y comunicar, debido a que se encuentra arraigado en la acción, la experiencia y la participación en un contexto específico (Nonaka, 1994). Incluye elementos cognitivos tales como los puntos de vista, creencias, valores, ideales o las intuiciones, y elementos técnicos como el know-how y las habilidades que se aplican a un contexto específico (Nonaka, 1994; Kim et al., 2014). En este sentido, el conocimiento tácito al no ser fácilmente comunicable, requiere interacciones entre las personas, generalmente equipos de personas u organizaciones a lo largo del tiempo para poder ser aplicado y transferido (Alegre, 2004).

\section{LAS PRÁCTICAS DE GESTIÓN DEL CONOCIMIENTO}

Las PGC se refieren al conjunto de técnicas, estrategias y procesos que son adoptados por la organización para la creación, almacenamiento, transferencia y aplicación del conocimiento (Alavi y Leidner, 2001; Simanca et al., 2016). Con base en el trabajo de Alegre y Lapiedra (2005) se consideran cuatro PGC: las prácticas de creación de conocimiento, las prácticas de aprendizaje continuo, los sistemas de conocimiento y retroalimentación, y la gestión de las competencias individuales de los empleados.

\section{Prácticas de creación de conocimiento}

De acuerdo con Lee y Choi (2003): “la creación de conocimiento se relaciona positivamente con la creatividad de las organizaciones, que está positivamente relacionada con el desempeño de la organización”. Las prácticas de creación de conocimiento comprenden los procesos a través de los cuales nuevos conocimientos son adquiridos y desarrollados o se reemplazan los existentes, resultando disponibles para su uso por parte de la organización (Alavi y Leidner, 2001). En este sentido, una estrategia capaz de aumentar la eficiencia de la GC y apoyar la transferencia de conocimientos entre los individuos y las empresas, es la codificación, 
mediante la transformación de conocimiento tácito en conocimiento codificado, es posible mejorar el acceso y la aplicación de conocimientos (Grover y Davenport, 2001; Massingham, 2014). Otro factor clave para la creación de conocimiento es el intercambio de conocimientos, mediante el cual los individuos comparten ideas, información relevante y experiencias organizativas con otros (Lee y Choi, 2003). El proceso de intercambio de conocimientos ha sido considerado como un componente clave de los sistemas de GC y uno de los elementos más importantes de los comportamientos creativos de la organización (Alavi y Leidner, 2001; Fullwood y Rowley, 2017). Teniendo en cuenta las consideraciones anteriores, se plantea la primera hipótesis de estudio: $H_{1}$ : Las prácticas de creación de conocimiento influencian la creatividad organizacional

\section{Prácticas de aprendizaje continuo}

Otro factor clave de éxito en el entorno competitivo actual es la capacidad de las organizaciones para aprender (Ahmed et al., 1999). Los procesos de aprendizaje, se llevan a cabo mediante la internalización del conocimiento, entendidido como la creación de nuevo conocimiento tácito a partir de conocimiento explícito, que resulta fundamental para el desarrollo de pensamientos novedosos y la generación de ideas (Shahzad et al., 2016). Mediante prácticas como formación, capacitación y tutorías, se adquieren nuevos conocimientos que pueden ser aplicados para la toma de deciciones o intercambiados con otras personas de la organización (Lee y Choi, 2003).

Las investigaciones han encontrado que el aprendizaje organizacional es esencial para la creatividad (Gong et al., 2009), en la medida en que inspira nuevos conocimientos e ideas, aumenta la capacidad de entenderlas y aplicarlas, intensifica el intercambio de conocimientos (Yeh et al., 2012), contribuye a la inteligencia organizacional, ayuda a identificar las oportunidades existentes, anticipando y comprendiendo las necesidades del cliente, las fortalezas y debilidades de sus rivales y los desarrollos tecnológicos disponibles, orientando sus capacidades a la innovación organizativa (García et al., 2012). Por lo tanto, es fundamental una cultura organizacional que lleve a los miembros de la organización a tener una permanente búsqueda de la mejora continua y un clima propicio para el aprendizaje (Ahmed et al., 1999; Verreynne et al., 2016). Con base en lo expuesto anteriormente, se plantea la segunda hipótesis de estudio: $H_{2}$ : Las prácticas de aprendizaje continuo inciden sobre la creatividad organizacional

\section{Sistemas de conocimiento y retroalimentación}

Los sistemas de conocimiento permiten la generación de nuevas ideas ya que crean redes de conocimiento que comparten información y resuelven problemas, ayudan a capturar y procesar integralmente la información, y establecen recursos dedicados para un propósito específico como la creación de proyectos o departamentos de investigación y desarrollo (Alavi y Leidner, 2001; Mao et al., 2016). Las actividades de control y supervisión de estos proyectos puede tener un impacto significativo sobre la creatividad cuando cuentan con objetivos generales claramente definidos y existe una frecuente retroalimentación entre el grupo de trabajo (Mainemelis et al., 2015; Amabile, 1997). Adicionalmente, las PGC que consisten en la retroalimentación frecuente y constructiva fomentan la creatividad mediante el fortalecimiento de la motivación intrínseca de los empleados, ya que les proporciona estándares para evaluar su propio trabajo, dirige la atención a la tarea en cuestión, fomenta una orientación al aprendizaje y el desarrollo, y facilita la obtención de habilidades y estrategias creativas (Zhou y George, 2001). Estas consideraciones son la base para la tercera hipótesis: $\mathrm{H}_{3}$ : Los sistemas de conocimiento y retroalimentación inciden en la creatividad organizacional

\section{Gestión de las competencias individuales de los empleados}

El sistema cultural y de recompensas de una organización puede tener un impacto significativo sobre la creatividad (Shalley y Gilson, 2004). El reconocimiento de las ideas creativas de los empleados es un motivador intrínseco que opera como soporte de la creatividad, y según Amabile (1997) la motivación intrínseca prevalece sobre las motivaciones extrínsecas, por ende, los sistemas de recompensa y retroalimentación sobre el trabajo promueven un entorno organizacional donde se reconocen las competencias y logros de los empleados, un aumento de la autonomía de trabajo, y mayores oportunidades de crecimiento profesional. En este punto, juega un rol importante el área de recursos humanos (Alegre y Lapiedra, 2005; Miterev et al., 2016). Con base en lo expuesto se plantea la cuarta y última hipótesis de investigación. En la Fig. 1 se muestra el modelo de hipótesis planteado: $H_{4}$ : Las prácticas de gestión de las competencias individuales de los empleados tienen incidencia sobre la creatividad de las organizaciones.

\section{METODOLOGÍA}

Se presentan en primer lugar las características de la muestra considerada, seguido por las escalas de medida empleadas y se finaliza con el análisis de datos por medio de pruebas de fiabilidad, variables de control y test del efecto mediador. 


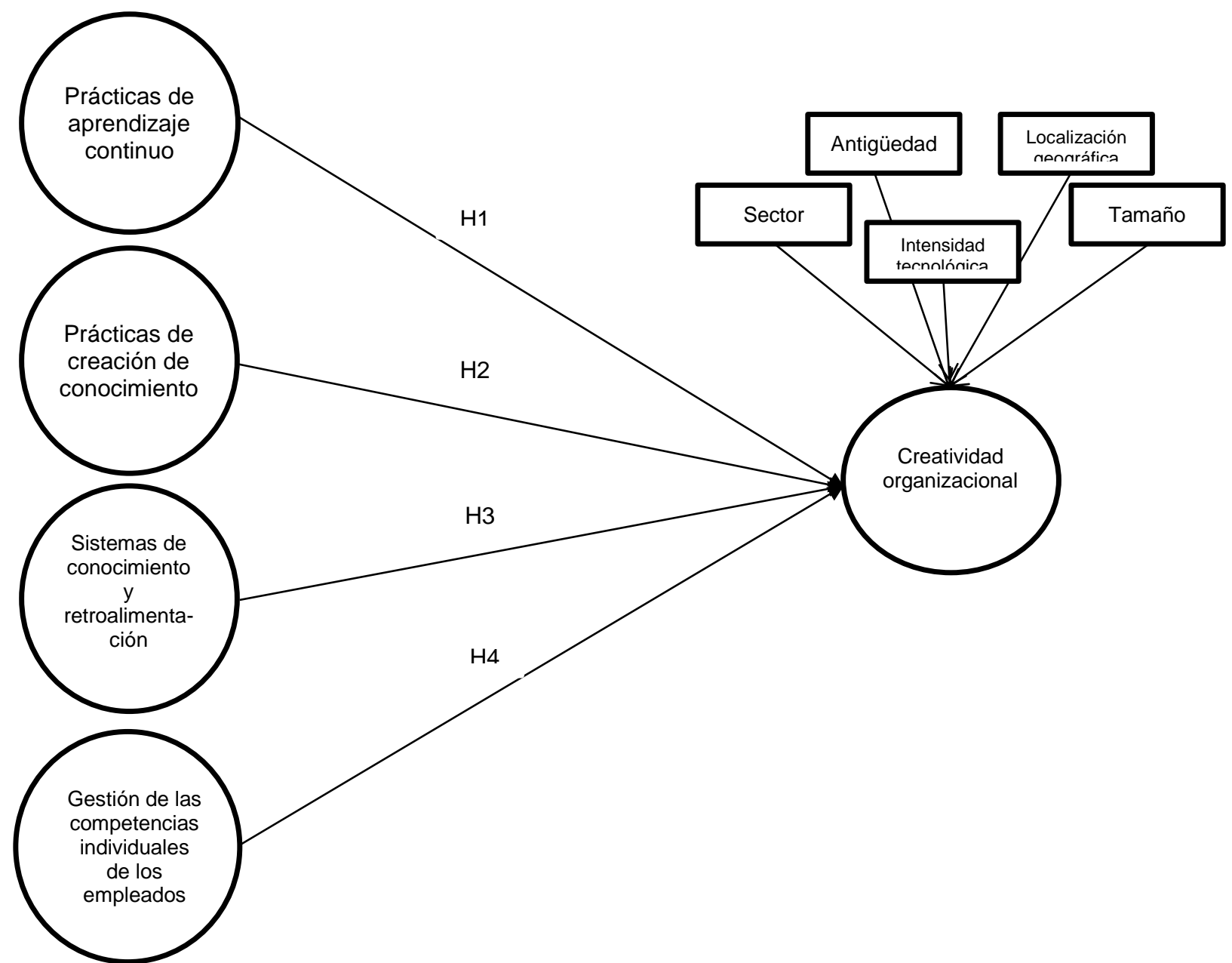

Fig. 1: Modelo de hipótesis

\section{Muestra}

El tamaño de la muestra fueron 160 empresas con representatividad a nivel nacional, de diferentes sectores de la economía, con una cuota importante de empresas dedicadas a actividades profesionales, científicas y técnicas; actividades de atención de la salud humana y de asistencia social; actividades industriales y educación (ver Tabla 1). De otra parte, el trabajo de campo se realizó en agosto de 2015, mediante el envío del cuestionario por correo electrónico al personal directivo, y las 160 respuestas válidas permitieron una potencia de prueba satisfactoria superior al $80 \%$ (Cohen, 1988).

\section{Escalas de medida}

En la tabla 2, se presentan los cinco constructos considerados y cada uno de los ítems de las escalas, para la medición de las PGC se consideraron cuatro constructos con 14 ítems de acuerdo con la escala elaborada por Alegre y Lapiedra (2005), compuesta por tres ítems para los constructos de prácticas de aprendizaje continuo y sistemas de conocimiento y retroalimentación, y cuatro para los constructos de prácticas de creación de conocimiento y gestión de las competencias individuales de los empleados. Los once ítems del constructo de creatividad organizacional fueron tomados de la escala propuesta en el trabajo de Zhou y George (2001). Se empleó una escala de Likert con rango desde muy inferior (1) hasta muy superior (5) en relación con sus competidores.

\section{Variables de control}

Con el fin de evitar distorsiones en los resultados, se consideraron cinco variables de control que son consideradas en estudios previos (Kim et al., 2012; Carmona et al., 2017), a saber: 1) tamaño, donde "1" representa las micro, "2" pequeñas, "3" medianas y "4" grandes empresas; 2) sector dummy, que toma valor "0" si es una empresa del sector manufacturero y "1" si es una empresa del sector servicios; 3) localización geográfica, es una variable dummy que toma valor "1" si es una empresa ubicada en Antioquia y Bogotá, y " 0 " si es una empresa ubicada en una región diferente a Antioquia y Bogotá (las muestras incluyen empresas de diferentes regiones de Colombia y en Antioquia y Bogotá se concentra el 70,86 \% de inversión en I+D y se encuentran las instituciones más importantes del Sistema Nacional de Ciencia Tecnología e Innovación, lo 
cual condiciona temas de innovación y creatividad; 4) antigüedad, medida como el logaritmo natural del número de años de existencia de la empresa; y 5) intensidad tecnológica y de conocimiento, que en el caso del sector manufacturero "0" agrupa empresas de baja y media - baja intensidad de tecnología y el "1" empresas de media alta y alta intensidad de tecnología; para el caso de sector servicios "0" agrupa empresas de baja intensidad de conocimiento y " 1 " empresas de alta intensidad de conocimiento.

Tabla 1: Características de las empresas de la muestra

\begin{tabular}{|c|c|c|}
\hline Sector & Frecuencia & Porcentaje \\
\hline Actividades profesionales, científicas y técnicas & 45 & $28,1 \%$ \\
\hline Actividades de atención de la salud humana y de asistencia social & 20 & $12,5 \%$ \\
\hline Educación & 13 & $8,1 \%$ \\
\hline Comercio & 12 & $7,5 \%$ \\
\hline Elaboración de productos alimenticios & 9 & $5,6 \%$ \\
\hline Actividades de servicios administrativos y de apoyo & 8 & $5,0 \%$ \\
\hline Información y comunicaciones & 8 & $5,0 \%$ \\
\hline Transporte y almacenamiento & 6 & $3,8 \%$ \\
\hline Actividades financieras y de seguros & 4 & $2,5 \%$ \\
\hline Alojamiento y servicios de comida & 4 & $2,5 \%$ \\
\hline Fabricación de maquinaria y equipo n.c.p. & 3 & $1,9 \%$ \\
\hline Fabricación de productos de caucho y de plástico & 3 & $1,9 \%$ \\
\hline Fabricación de sustancias y productos químicos & 3 & $1,9 \%$ \\
\hline Distribución de agua, evacuación y tratamiento de aguas residuales & 2 & $1,3 \%$ \\
\hline Fabricación de productos textiles & 2 & $1,3 \%$ \\
\hline Otras industrias manufactureras & 2 & $1,3 \%$ \\
\hline Actividades artísticas, de entretenimiento y recreación & 1 & $0,6 \%$ \\
\hline Actividades de impresión y actividades de servicios relacionados con la impresión & 1 & $0,6 \%$ \\
\hline Actividades inmobiliarias & 1 & $0,6 \%$ \\
\hline Confección de prendas de vestir & 1 & $0,6 \%$ \\
\hline Curtido y recurtido de cueros & 1 & $0,6 \%$ \\
\hline Elaboración de bebidas & 1 & $0,6 \%$ \\
\hline Fabricación de aparatos y equipo eléctrico & 1 & $0,6 \%$ \\
\hline Fabricación de muebles, colchones y somieres & 1 & $0,6 \%$ \\
\hline Fabricación de otros productos minerales no metálicos & 1 & $0,6 \%$ \\
\hline Fabricación de papel, cartón y productos de papel y cartón & 1 & $0,6 \%$ \\
\hline Fabricación de productos elaborados de metal, excepto maquinaria y equipo & 1 & $0,6 \%$ \\
\hline Fabricación de productos farmacéuticos & 1 & $0,6 \%$ \\
\hline Fabricación de productos metalúrgicos básicos & 1 & $0,6 \%$ \\
\hline Instalación, mantenimiento y reparación especializado de maquinaria y equipo & 1 & $0,6 \%$ \\
\hline $\begin{array}{l}\text { Transformación de la madera y fabricación de productos de madera y de corcho, } \\
\text { excepto muebles }\end{array}$ & 1 & $0,6 \%$ \\
\hline Otras actividades de servicios & 1 & $0,6 \%$ \\
\hline Total & 160 & $100 \%$ \\
\hline
\end{tabular}


Tabla 2: Ítems de las escalas

\begin{tabular}{|c|c|}
\hline \multicolumn{2}{|r|}{ Prácticas de gestión del conocimiento } \\
\hline \multicolumn{2}{|c|}{ Prácticas de creación de conocimiento } \\
\hline$P G C 1$ & Sistemas para codificar y hacer explícito el conocimiento \\
\hline PGC2 & Mecanismos para incentivar a los colaboradores a compartir su conocimiento \\
\hline PGC3 & $\begin{array}{l}\text { Metodologías para propiciar el intercambio de conocimiento como las comunidades de práctica, los } \\
\text { círculos de calidad, los grupos de mejoramiento, o sus equivalentes. }\end{array}$ \\
\hline PGC4 & $\begin{array}{l}\text { Sistema de diseminación de información para empleados, clientes y proveedores de acuerdo con } \\
\text { sus necesidades. }\end{array}$ \\
\hline \multicolumn{2}{|c|}{ Prácticas de aprendizaje continuo } \\
\hline PGC5 & Plan de formación que incentiva el aprendizaje continuo del empleado \\
\hline PGC6 & $\begin{array}{l}\text { Formación general para los empleados, quienes posteriormente aplican lo aprendido en sus } \\
\text { actividades habituales de trabajo }\end{array}$ \\
\hline$P G C 7$ & $\begin{array}{l}\text { Sistema de mejoramiento continuo que permite mejorar los procesos que han alcanzado los } \\
\text { estándares de calidad establecidos. }\end{array}$ \\
\hline \multicolumn{2}{|c|}{ Sistemas de conocimiento y retroalimentación } \\
\hline PGC8 & Sistema de captura y procesamiento integral de la información de los diferentes procesos \\
\hline PGC9 & Mecanismos de control y revisión de los proyectos de innovación \\
\hline PGC10 & $\begin{array}{l}\text { De los proyectos de innovación se obtiene retroalimentación útil para el desarrollo de nuevos } \\
\text { proyectos }\end{array}$ \\
\hline \multicolumn{2}{|c|}{ Gestión de las competencias individuales de los empleados } \\
\hline PGC11 & La dirección reconoce y comunica formalmente los logros de sus colaboradores \\
\hline PGC12 & Sistemas para medir y evaluar las competencias de los empleados \\
\hline PGC13 & $\begin{array}{l}\text { Sistema de remuneración y promoción de los empleados que influye en el desarrollo de sus } \\
\text { competencias, ideas y conocimientos }\end{array}$ \\
\hline PGC14 & Técnicas de referenciación o benchmarking para la mejora de las competencias de los empleados \\
\hline \multicolumn{2}{|r|}{ Creatividad } \\
\hline C1 & Sugerir nuevas formas para alcanzar metas y objetivos. \\
\hline C2 & Presentar ideas nuevas y prácticas para mejorar el desempeño de la organización. \\
\hline C3 & Investigar sobre nuevas tecnologías, procesos, o productos. \\
\hline C4 & Sugerir nuevas maneras para incrementar la calidad. \\
\hline C5 & Ser una buena fuente de ideas creativas. \\
\hline C6 & No tener temor a tomar riesgos. \\
\hline C7 & Promover e impulsar las ideas propias ante otras personas. \\
\hline C8 & Mostrar creatividad en el trabajo cuando se les da la oportunidad de hacerlo. \\
\hline C9 & Desarrollar planes y cronogramas adecuados para la implementación de nuevas ideas. \\
\hline C10 & Generar a menudo ideas innovadoras. \\
\hline C11 & Proponer soluciones creativas a problemas reales. \\
\hline C12 & Abordar a menudo los problemas con un enfoque nuevo y original. \\
\hline C13 & Sugerir nuevas maneras de hacer su trabajo. \\
\hline
\end{tabular}

\section{Análisis de datos}

La valoración del modelo estructural se obtuvo mediante pruebas de fiabilidad y validez para los cinco constructos considerados y las variables de control. Además, se emplearon ecuaciones estructurales por el método de mínimos cuadrados, para comprobar el efecto mediador de las diferentes trayectorias. 


\section{Fiabilidad y validez}

Con el fin de verificar la consistencia interna de las variables, se realizó un análisis de fiabilidad individual y fiabilidad compuesta. Se constató que la fiabilidad individual de todos los ítems tenía una carga factorial superior a 0,7. Adicionalmente, se validó la fiabilidad compuesta de los constructos puesto que la carga factorial fue superior a 0,7 para cada uno de ellos (Chin, 2010). Así mismo, se evaluó la validez convergente de acuerdo con el índice de varianza extraída superior a 0,5 (Fornell y Larcker, 1981) lo que significa que el conjunto de indicadores representan los constructos del modelo. La información obtenida se presenta en la Tabla 3, para los datos con asterisco $\left(^{*}\right)$ los valores $p \leq 0,001$

Tabla 3: Fiabilidad y validez convergente

\begin{tabular}{|c|c|c|c|c|c|}
\hline Constructo & Carga estandarizada & Error estándar & Valor $t$ & $\begin{array}{l}\text { Fiabilidad } \\
\text { compuesta }\end{array}$ & Índice de varianza extraída \\
\hline \multicolumn{4}{|c|}{ Prácticas de creación de conocimiento } & 0,89 & 0,67 \\
\hline PGC1 & $0,82^{*}$ & 0,02 & 33,24 & & \\
\hline PGC2 & $0,85^{\star}$ & 0,02 & 47,80 & & \\
\hline PGC3 & $0,83^{*}$ & 0,03 & 31,65 & & \\
\hline PGC4 & $0,76^{*}$ & 0,04 & 20,43 & & \\
\hline \multicolumn{4}{|c|}{ Prácticas de aprendizaje continuo } & 0,94 & 0,83 \\
\hline PGC5 & $0,93^{*}$ & 0,01 & 101,17 & & \\
\hline PGC6 & $0,92^{*}$ & 0,01 & 68,79 & & \\
\hline PGC7 & $0,89^{*}$ & 0,02 & 56,25 & & \\
\hline \multicolumn{4}{|c|}{ Sistemas de conocimiento y retroalimentación } & 0,91 & 0,77 \\
\hline PGC8 & $0,77^{*}$ & 0,04 & 20,98 & & \\
\hline PGC9 & $0,93^{*}$ & 0,01 & 100,61 & & \\
\hline PGC10 & $0,92^{*}$ & 0,01 & 79,14 & & \\
\hline \multicolumn{4}{|c|}{ Gestión de las competencias individuales de los empleados } & 0,92 & 0,73 \\
\hline PGC11 & $0,82^{*}$ & 0,02 & 38,05 & & \\
\hline PGC12 & $0,85^{\star}$ & 0,02 & 36,39 & & \\
\hline PGC13 & $0,87^{*}$ & 0,03 & 32,49 & & \\
\hline PGC14 & $0,88^{*}$ & 0,02 & 49,36 & & \\
\hline \multicolumn{4}{|l|}{ Creatividad } & 0,97 & 0,72 \\
\hline C1 & $0,78^{*}$ & 0,03 & 25,31 & & \\
\hline $\mathrm{C} 2$ & $0,81^{*}$ & 0,02 & 36,33 & & \\
\hline C3 & $0,79^{*}$ & 0,02 & 34,18 & & \\
\hline $\mathrm{C} 4$ & $0,79^{*}$ & 0,03 & 23,23 & & \\
\hline C5 & $0,87^{*}$ & 0,02 & 53,86 & & \\
\hline C6 & $0,81^{*}$ & 0,03 & 28,68 & & \\
\hline C7 & $0,86^{*}$ & 0,02 & 52,09 & & \\
\hline C8 & $0,85^{*}$ & 0,02 & 46,23 & & \\
\hline $\mathrm{C9}$ & $0,85^{*}$ & 0,02 & 44,43 & & \\
\hline C10 & $0,89^{*}$ & 0,01 & 63,32 & & \\
\hline C11 & $0,87^{*}$ & 0,01 & 62,64 & & \\
\hline C12 & $0,89^{*}$ & 0,01 & 68,68 & & \\
\hline $\mathrm{C} 13$ & $0,91^{*}$ & 0,01 & 91,98 & & \\
\hline Tamaño & 1 & 0 & 0 & 1 & 1 \\
\hline Sector & 1 & 0 & 0 & 1 & 1 \\
\hline Localización & 1 & 0 & 0 & 1 & 1 \\
\hline Antigüedad & 1 & 0 & 0 & 1 & 1 \\
\hline Intensidad & 1 & 0 & 0 & 1 & 1 \\
\hline
\end{tabular}

El análisis factorial confirmatorio permitió constatar la validez discriminante, dado que la raíz cuadrada del índice de varianza extraída supera las correlaciones entre los distintos constructos (Chin, 1998). En la Tabla 4 se observa que esta condición se cumple en todos los casos. Bajo este concepto se encuentra que todas las variables latentes cumplen con este criterio. Las variables latentes están más correlacionadas con sus indicadores que con el resto de las variables. 
Tabla 4: Validez discriminante

\begin{tabular}{|l|c|c|c|c|c|}
\hline \multicolumn{1}{|c|}{ Constructo } & 1 & 2 & 3 & 4 & 5 \\
\hline Prácticas de aprendizaje continuo & 0,91 & & & & \\
\hline Gestión de las competencias individuales & 0,67 & 0,86 & & & \\
\hline Prácticas de creación de conocimiento & 0,72 & 0,65 & 0,82 & & \\
\hline Creatividad & 0,58 & 0,66 & 0,57 & 0,85 & \\
\hline Sistemas de conocimiento y retroalimentación & 0,53 & 0,72 & 0,64 & 0,56 & 0,88 \\
\hline
\end{tabular}

\section{Test del efecto mediador}

Con el fin de comprobar la mediación de las variables se realizó un análisis de ecuaciones estructurales por el método mínimos cuadrados, mediante un remuestreo o boostrap de 5000 submuestras, que permitió conocer los valores t y los errores estándar de los coeficientes de las trayectorias consideradas y establecer la significancia estadística (Henseler et al., 2009). Los resultados presentados en la Tabla 5 muestran que dos de los cuatro constructos del modelo influyen positivamente sobre la creatividad organizacional. Se confirma que las prácticas de aprendizaje continuo tienen una relación significativa con la creatividad organizacional, es decir, se acepta la $H_{2}(\beta=0,19$; valor $t=2,54)$. Además, se encuentra que la gestión de las competencias individuales de los empleados influye significativamente sobre la creatividad de la organización, comprobándose la $H_{4}(\beta=0,33$; valor $t=4,11)$. Sin embargo, no se observa una influencia significativa entre la creatividad organizacional y las prácticas de creación de conocimiento, así como con los sistemas de conocimientos y retroalimentación, de igual manera, no se logra evidenciar su efecto sobre las variables de control. Por lo tanto, los resultados sugieren que las prácticas de GC que se encuentran relacionadas con los procesos de formación y aprendizaje y el desarrollo de competencias, tienen un efecto significativo en la generación de ideas creativas. Para los coeficientes con asterisco $\left(^{*}\right)$ los valores $p \leq 0,05 \mathrm{y}$ doble asterisco $\left({ }^{* *}\right)$ los valores $p \leq 0,001$

Tabla 5: Resultados de las ecuaciones estructurales

\begin{tabular}{|l|c|c|}
\hline \multicolumn{1}{|c|}{ Trayectoria } & Coeficiente & Valor $t$ \\
\hline Prácticas de creación de conocimiento $\rightarrow$ Creatividad & 0,14 & 1,95 \\
\hline Prácticas de aprendizaje continuo $\rightarrow$ Creatividad & $0,19^{*}$ & 2,54 \\
\hline Sistemas de conocimiento y retroalimentación $\rightarrow$ Creatividad & 0,13 & 1,52 \\
\hline Gestión de las competencias individuales $\rightarrow$ Creatividad & $0,33^{* *}$ & 4,11 \\
\hline Edad $\rightarrow$ Creatividad & $-0,12$ & 2,42 \\
\hline Tamaño $\rightarrow$ Creatividad & $-0,05$ & 1,09 \\
\hline Intensidad tecnológica $\rightarrow$ Creatividad & $-0,04$ & 0,95 \\
\hline Región $\rightarrow$ Creatividad & 0,01 & 0,18 \\
\hline Sector $\rightarrow$ Creatividad & 0,00 & 0,07 \\
\hline
\end{tabular}

\section{CONCLUSIONES}

El presente artículo contribuye a identificar la manera como el conocimiento puede ser gestionado dentro de las organizaciones para promover la generación de ideas nuevas y útiles, reconociendo que la puesta en marcha de prácticas concretas de gestión del conocimiento fomenta el ambiente organizacional, proporciona recursos y desarrolla procesos que promueven la creatividad y facilitan el proceso de innovación. Por lo tanto, resulta fundamental el desarrollo de una cultura organizacional que fomente la generación de nuevas ideas y maneras de hacer las cosas, poniendo especial atención a los procesos de aprendizaje organizacional, tales como la realización de programas de formación y el desarrollo de sistemas de mejora continua, así como las prácticas de gestión de las competencias de los empleados y los sistemas de remuneración que buscan medir, mejorar, comunicar y reconocer sus competencias. Las organizaciones en la actual economía tienen la obligación de encontrar estrategias que le permitan potenciar su creatividad para la solución de problemas, nuevas maneras de hacer las cosas, el desarrollo de nuevas ideas y formas de alcanzar sus metas y objetivos. El artículo reconoce que la habilidad de las organizaciones para crear, almacenar, transferir y aplicar conocimientos resulta fundamental para tal fin. De ahí la importancia de evaluar y comprender la posición del conocimiento en las organizaciones para la puesta en marcha de prácticas de gestión del conocimiento y los resultados que pueden tener en la generación de ideas nuevas y útiles, elementos claves para la supervivencia y obtención de ventajas competitivas a largo plazo. 


\section{AGRADECIMIENTOS}

Este artículo es producto del proyecto de investigación "Determinantes de la capacidad de absorción empresarial", financiado por el CODI- Comité para el Desarrollo de la Investigación- de la Universidad de Antioquia, 2013-2015.

\section{REFERENCIAS}

Adelstein, J. y Clegg, S., And rewind! Recycling discourses of knowledge work and knowledge society, Management \& Organizational History, 9 (1), 3-25 (2014)

Ahmed, P.; A.Y.E. Loh, y M. Zairi, Cultures for continuous improvement and learning, doi: 10.1080/0954412997361, Total Quality Management, 10 (4-5), 426-434 (1999)

Alavi, M. y D.E. Leidner, Review: knowledge management and knowledge management systems: conceptual foundations and research issues, doi: 10.2307/3250961, Management Information Systems Quaterly, 25 (1), 107-136 (2001)

Alegre, J., La gestión del conocimiento como motor de la innovación: lecciones de la industria de alta tecnología para la empresa, 158, Publicacions de la Universitat Jaume I, Castellón de la Plana, España (2004)

Alegre, J., y R. Lapiedra, Gestión del conocimiento y desempeño innovador: un estudio del papel mediador del repertorio de competencias distintivas, Cuadernos de Economía y Dirección de la Empresa (23), 117-138 (2005)

Amabile, T.M., A model of creativity and innovation in organizations, Research in organizational behavior, 10 (1), 123-167 (1988)

Amabile, T.M., Motivating creativity in organizations: on doing what you love and loving what you do, doi: 10.2307/41165921, California Management Review, 40 (1), 39-58 (1997)

Amabile, T.M. y R. Conti, Changes in the Work Environment for Creativity during Downsizing, doi: 10.2307/256984, Academy of Management Journal, 42 (6), 630-640 (1999)

Anderson, N.; K. Potočnik y Zhou, J., Innovation and Creativity in Organizations: A State-of-the-Science Review, Prospective Commentary, and Guiding Framework, doi: 10.1177/0149206314527128, Journal of Management, 40 (5), 1297-1333 (2014)

Barney, J., Firm resources and sustained competitive advantage, doi: 10.1177/014920639101700108, Journal of management, 17 (1), 99-120 (1991)

Carmona-Osorio, C.; Ángel-Gallego, S. y Arias-Pérez, J., Strategic orientation and strategies to manage organisational knowledge and creativity, Academia Revista Latinoamericana de Administración, 30 (3), 312$327(2017)$

Chin, W.W., The partial least squares approach to structural equation modeling, in Modern methods for business research by G.A. Marcoulides, pp. 295-336, Lawrence Erlbaum Associates Publishers, Mahwah, USA (1998)

Chin, W.W., How to write up and report PLS analyses, in Handbook of partial least squares: Concepts, methods and applications by V. Esposito, W.W. Chin; J. Henseler y H. Wang, pp. 655-690, Springer Science \& Business Media, Berlin, Alemania (2010)

Cohen, J., Statistical power analysis for the behavioral sciences, $2^{\text {nd }}$ Ed. Academic Press, New York, USA (1988)

Davenport, T.H. y L. Prusak, Working Knowledge: How Organizations Manage What They Knowledge, Harvard Business Press, Boston, USA (1998)

Donate, M.J., y J.D. Sánchez de Pablo, The role of knowledge-oriented leadership in knowledge management, Journal of Business Research, (68), 360-370 (2015)

Fornell, C. y D.F. Larcker, Evaluating structural equation models with unobservable variables and measurement error, doi: 10.2307/3151312, Journal of Marketing Research, 18 (3), 39-50 (1981) 
Fullwood, R. y Rowley, J., An investigation of factors affecting knowledge sharing amongst UK academics, Journal of Knowledge Management, (just-accepted), 1-34 (2017)

García-Quevedo, J., Mas-Verdú, F. y Montolio, D., What types of firms acquire knowledge intensive services and from which suppliers? Technology Analysis \& Strategic Management, 25 (4), 473-486 (2013)

García, V.J.; M.M. Jiménez y L. Gutiérrez, Transformational leadership influence on organizational performance through organizational learning and innovation, Journal of Business Research, 65 (7), 1040-1050 (2012)

Gong, Y.; J.C. Huang y J.L. Farh, Employee learning orientation, transformational leadership, and employee creativity: The mediating role of employee creative self-efficacy, Academy of management Journal, 52 (4), 765-778 (2009)

Grant, R.M., Toward a Knowledge-Based Theory of the Firm, Strategic Management Journal, 17 (Winter Special Issue), 109-122 (1996)

Grover, V. y T.H. Davenport, General Perspectives on Knowledge Management: Fostering a Research Agenda, doi: 10.1080/07421222.2001.11045672, Journal of Management Information Systems, 18 (1), 5-21 (2001)

Henseler, J.; C.M. Ringle y R.R. Sinkovics, The use of partial least squares path modeling in international marketing. Advances in International Marketing (AIM), in New Challenges to International Marketing Advances in International Marketing by R.R. Sinkovics y P.N. Ghauri, 20, 277-320, Emerald Group Publishing Limited (2009)

Kim, N.; Im, S. y Slater, S. F., Impact of knowledge type and strategic orientation on new product creativity and advantage in high-technology firms, Journal of Product Innovation Management, 30 (1), 136-153 (2013)

Kim, T. H.; Lee, J. N.; Chun, J. U. y Benbasat, I., Understanding the effect of knowledge management strategies on knowledge management performance: A contingency perspective. Information \& management, 51 (4), 398-416 (2014)

Lee, V. H.; Foo, A. T. L.; Leong, L. Y. y Ooi, K. B., Can competitive advantage be achieved through knowledge management? A case study on SMEs, Expert Systems with Applications, 65, 136-151 (2016)

Lee, H. y B. Choi, Knowledge management enablers, processes, and organizational performance: an integrative view and empirical examination, doi: 10.1080/07421222.2003.11045756, Journal of Management Information Systems, 20 (1), 179-288 (2003)

Mainemelis, C.; R. Kark y O. Epitropaki, Creative Leadership: A Multi-Context Conceptualization, doi: 10.1080/19416520.2015.1024502, The Academy of Management Annals, 9 (1), 393-482 (2015)

Mao, H.; Liu, S.; Zhang, J. y Deng, Z., Information technology resource, knowledge management capability, and competitive advantage: the moderating role of resource commitment. International Journal of Information Management, 36 (6), 1062-1074 (2016)

Massingham, P., An evaluation of knowledge management tools: Part 1-managing knowledge resources, Journal of Knowledge Management, 18 (6), 1075-1100 (2014)

Miterev, M.; Engwall, M. y Jerbrant, A., Exploring program management competences for various program types, International Journal of Project Management, 34 (3), 545-557 (2016)

Nonaka, I., A Dynamic Theory of Organizational Knowledge Creation, doi: 10.1287/orsc.5.1.14, Organization science, 5 (1), 14-37 (1994)

Nonaka, I. y H. Takeuchi, The knowledge-creating company: How Japanese companies create the dynamics of innovation, pp. 304, Oxford university press, Nueva York, USA (1995)

Phipps, S.T.A. y L.C. Prieto, Knowledge is power? An inquiry into knowledge management, Its effects on individual creativity, and the moderating role of an entrepreneurial mindset, Academy of Strategic Management Journal, 11 (1), 43-58 (2012)

Polanyi, M., The Tacit Dimension, pp. 108, Routledge and Kegan Paul, Londres, Inglaterra (1966) 
Rhee, Y.W. y Choi, J.N., Knowledge management behavior and individual creativity: Goal orientations as antecedents and in-group social status as moderating contingency, Journal of Organizational Behavior, 38 (6), 813-832 (2017)

Simanca, M.; Montoya, L. y Bernal, C. A., Gestión del Conocimiento en Cadenas Productivas: El Caso de la Cadena Láctea en Colombia, Información Tecnológica, 27 (3), 93-106 (2016)

Shahzad, K.; S.U. Bajwa; A.F.I Siddiqi; F. Ahmid y A. Raza, Integrating knowledge management (KM) strategies and processes to enhance organizational creativity and performance: an empirical investigation, doi: 10.1108/JM2-07-2014-0061, Journal of Modelling in Management, 154-179 (2016)

Shalley, C. y L.L. Gilson, What leaders need to know: A review of social and contextual factors that can foster or hinder creativity, The Leadership Quarterly, 15 (1), 33-53 (2004)

Sigala, M. y Chalkiti, K., Knowledge management, social media and employee creativity, International Journal of Hospitality Management, 45, 44-58 (2015)

Vásquez-Rizo, F.E. y Gabalán-Coello, J., Información y ventaja competitiva. Coexistencia exitosa en las organizaciones de vanguardia, El Profesional de la Información, 24 (2), 149-156 (2015)

Van de Ven, A., Central problems in the management of innovation, doi: $10.1287 / \mathrm{mnsc} .32 .5 .590$, Management science, 32 (5), 590-607 (1986)

Verreynne, M. L.; Hine, D.; Coote, L. y Parker, R., Building a scale for dynamic learning capabilities: The role of resources, learning, competitive intent and routine patterning, Journal of Business Research, 69 (10), 4287 4303 (2016)

Von Krogh, G.; K. Ichijo y I. Nonaka, Enabling Knowledge Creation: How to Unlock the Mystery of Tacit Knowledge and Release the Power of Innovation, pp. 304, Oxford University Press, New York, USA (2000)

Yeh, Y.C.; Y.L. Yeh y Y.H. Chen, From knowledge sharing to knowledge creation: A blended knowledgemanagement model for improving university students' creativity, Thinking Skills and Creativity, 7(3), 245-257 (2012)

Zhou, J. y J. M., George, When job dissatisfaction leads to creativity: Encouraging the expression of voice, Academy of Management Journal, 44 (4), 682-696 (2001) 